\title{
Seeking for sensory differentiated olive oils? The urge to preserve old autochthonous olive cultivars
}

\author{
Nuno Rodrigues $^{\mathrm{a}}$, Susana Casal ${ }^{\mathrm{b}}$, António M. Peres ${ }^{\mathrm{a}, \mathrm{c}}$, Paula Baptista ${ }^{\mathrm{a}}$, José Alberto Pereira ${ }^{\mathrm{a}, *}$ \\ ${ }^{a}$ Centro de Investigação de Montanha (CIMO), ESA, Instituto Politécnico de Bragança, Campus de Santa Apolónia, 5300-253 Bragança, Portugal \\ ${ }^{\mathrm{b}}$ REQUIMTE, Laboratory of Bromatology and Hydrology, Faculty of Pharmacy, University of Porto, Rua de Jorge Viterbo Ferreira, 228, 4050-313 Porto, Portugal \\ ${ }^{\mathrm{c}}$ Laboratory of Separation and Reaction Engineering - Laboratory of Catalysis and Materials (LSRE-LCM), ESA, Instituto Politécnico de Bragança, Campus Santa \\ Apolónia, 5300-253 Bragança, Portugal
}

A R T I C L E I N F O

\section{Keywords:}

Olive heritage

Centenarian olive trees

Chemical characterization

Sensory attributes

Cultivar discrimination

\begin{abstract}
A B S T R A C T
Mediterranean olive heritage richness is poorly characterized. Olive oils from minor cultivars of NortheastPortugal (Lentisca, Madural, Rebolã, Redondal, Verdeal and Verdeal Transmontana) from centenarian trees were chemical and sensory characterized, aiming to identify autochthonous cultivars capable of producing differentiated olive oils. All oils, produced during two campaigns, were classified as extra virgin. $C v$. Redondal showed the highest oxidative stability (OS), total phenols, vitamin $\mathrm{E}$ and $\mathrm{C}_{18: 1} / \mathrm{C}_{18: 2}$. Contrary, $c v$. Madural presented the lowest OS and $\mathrm{C}_{18: 1} / \mathrm{C}_{18: 2}$ ratios, supporting the importance of fatty acids on OS, while $c v$. Verdeal had the lowest total phenols and vitamin E contents. Sensory notes of tomato, apple, dry fruits, fresh herbs, tomato leaves and cabbage were predominant on the oils of most cultivars, whilst some attributes were more specific, such as banana and kiwi (Madural), cherry and apricot (cvs. Lentisca and Madural). The chemical and sensory diversity enabled the statistical discrimination of all cultivars and harvesting years.
\end{abstract}

\section{Introduction}

Olive oil is a product of great importance in Mediterranean countries. It is also one of the pillars of the Mediterranean diet. Due to its composition, nutritional properties and organoleptic characteristics, olive oil is an ingredient present in most dishes made and consumed daily. The origin of the olive tree is not well defined but it is believed that it began in the Asia Minor region, from where it spread to the Mediterranean basin, and from there to almost all continents. Therefore, in this region the existence of ancient olive trees, millenarian and centenarian, represents a very important genetic heritage that needs to be characterized, preserved and valorized. The productivity of these old specimens is usually low and not economically competitive. However, the current market trends of high quality extra virgin olive oil (EVOO) valorizes differentiated products from traditional cultivars (produced in non-intensive systems with specific geographical origins), products rich in health promoting bioactive compounds, as well as products with differentiated sensorial characteristics (Bajoub et al., 2015; Del Monaco et al., 2015; Genovese, Yang, Linforth, Sacchi, \& Fisk, 2018; Reboredo-Rodríguez et al., 2016; Zago, Squeo, Bertoncini, Difonzo, \& Caponio, 2019). The combination of these characteristics is only found in traditional cultivars, and particularly in centennial and millennial specimens grown in low input systems with specific "terroirs" thus making this type of olive oil singular and unique in the market (Reboredo-Rodríguez et al., 2015). In this context, olive oils extracted from olives produced by old trees (centenarian or millenarian) could have a great interest and acceptance, producing differentiated oils that could be competitive in the national and international markets (Salimonti et al., 2013). Nevertheless, the information available on old olive trees is very scarce. It is generally established that an olive cultivar, and consequently its genetic information, is a determining factor for the composition, nutritional value and sensory characteristics of olive oils (Bajoub et al., 2015; Fernandes, Ellis, Gámbaro, \& BarreraArellano, 2018; Köseoğlu, Sevim, \& Kadiroğlu, 2016). Some works demonstrated that olive cultivars influence the overall chemical composition of the obtained oils (Bajoub et al., 2015; Garcia, Magalhães, Fregapane, Salvador, \& Paiva-Martins, 2012; Köseoğlu et al., 2016; Lukić et al., 2018; Xiang et al., 2017), whilst other demonstrated the influence of the cultivars in specific chemical families, as fatty acids (Krichene et al., 2010; Kritioti, Menexes, \& Drouza, 2018; ReboredoRodríguez et al., 2015), tocopherols (Beltrán et al., 2010; Blasi et al., 2018), phenolic compounds (Del Monaco et al., 2015; Blasi et al., 2018), and sterols (Krichene et al., 2010). Among these, some minor compounds are of major importance for the sensory attributes, like

\footnotetext{
* Corresponding author.

E-mail address: jpereira@ipb.pt (J.A. Pereira).
} 
polyphenols and volatiles, which are responsible for olive oil bitterness, pungency, and typical aroma (Aparicio-Ruiz, García-González, Morales, Lobo-Prieto, \& Romero, 2018; García-Vico, García-Rodríguez, Sanz, \& Pérez, 2017). According to Genovese et al. (2018), the flavor notes derived from volatile and phenolic compounds are the main features evaluated in the organoleptic assessment of virgin olive oils (VOO). However, the final sensory profile has a far more complex nature, being also influenced by the soil characteristics, climate, tree health, fruit maturation at the time of harvest, olive harvesting process, olive storage conditions, extraction process and storage method of olive oil prior to packaging (Genovese et al., 2018; Gerhardt et al., 2019; Lukić et al., 2018; Köseoğlu et al., 2016; Valli et al., 2019). Unfortunately, the cultivar effect on oils obtained from centenarian trees is not well documented, nor the "terroir" impacts. Trás-os-Montes is the second largest Portuguese olive-growing region, with a characteristic climate and soil that has contributed for the internationally recognized quality of the olive oils produced in this region. Also, a considerable number of ancient trees found in this region, some with more than 200 years, constitutes a great olive heritage that should be explored. These ancient trees could be considered a biodiversity reservoir of minor cultivars, unknown or poorly characterized. In this context, olive oils extracted from fruits of ancient trees were analyzed for chemical and sensory attributes over two consecutive crop years (2016 and 2017) in order to contribute for its exploitation and valorization as a way to preserve the olive heritage of Trás-os-Montes region. The chosen trees belong to six cultivars, four of them (Lentisca, Rebolã, Redondal and Verdeal) characterized for the first time in the present work, and the other two still poorly known (Madural and Verdeal Transmontana).

\section{Material and methods}

\subsection{Olive oils samples}

One olive grove with centenarian trees ( $>250$ years) was selected near Mirandela (N 41 $29^{\prime} 26.628^{\prime \prime}$; W $7^{\circ} 15^{\prime} 31.219^{\prime \prime}$ ), northeast of Portugal. Based on the tree appearance, structure and trunk thickness, 21 randomly trees were selected and marked. After morphological characterization of fruits and stones (see Supplementary Material Table S1), and with the help of experts from the Olive Producers Association of Trás-os-Montes and Alto Douro, the trees were identified as belonging to minor cultivars namely cvs. Lentisca ( 3 trees), Madural ( 3 trees), Rebolã ( 3 trees), Redondal ( 3 trees), Verdeal (2 trees) and Verdeal Transmontana (7 trees). From each tree, during two crop years (2016 and 2017), approximately three kilograms of fruits were manually collected. To overcome the influence of the maturity stage on the olive oil composition, harvest occurred when the fruits were between the maturity stage two and three, when the fruit epidermis presents red spots in less than half the olive (MI 2) and the fruit epidermis is red or purple in more than half the olive (MI 3). Therefore, harvests took place on different dates in each year to grant similar maturity stages, taking place on the 07 th and 08th November in 2016, and on the 13th and 14th November in 2017. All fruits were inspected in order to avoid injured fruits attacked by pests or infested by diseases to maintain the maximum quality of the oils. The fruits were processed in the first $24 \mathrm{~h}$ after harvest, in a pilot extraction plant with an Abencor analyzer (Comercial Abengoa S.A., Seville, Spain). The olive oils were put in $125 \mathrm{~mL}$ dark bottles and stored in the dark at room temperature. All the assays were carried out in triplicate.

\subsection{Evaluation of quality parameters}

Olive oils were analyzed according to the methodologies described by the European Union standard methods (Annexes II and IX in European Community Regulation EEC/2568/91 from 11th July and amendments). Free acidity (FA, in \% of oleic acid), peroxide value (PV, in $\mathrm{mEqO}_{2} / \mathrm{Kg}$ ), and the specific coefficients of extinction at $232 \mathrm{~nm}$ and
$270 \mathrm{~nm}\left(\mathrm{~K}_{232}\right.$ and $\left.\mathrm{K}_{270}\right)$ were determined. A sensory panel comprising 8 trained panelists evaluated all olive oil samples, following the abovementioned European Community Regulation. The descriptive profile was assessed using a test sheet with some modification following the recommendations of the International Olive Council (IOC) (COI/T.20/ Doc. at the. 22 November 2005). The olfactory intensities were graded using a continuous scale ranging from 0 (no perceived sensation) to 10 (maximum intensity of perceived sensation), being assessed the intensity of fruity (mature or green), fruits sensations, herbaceous sensations and harmony. The intensities of the gustatory-retronasal attributes were graded using a similar scale, being evaluated the intensity of fruity (mature or green), sweet, bitter, pungent, fruits sensations, herbaceous sensations and harmony. To establish the sensory profile and to not influence the panelists, blank lines for the identification of possible sensory descriptors were included in the test sheet, without any reference to the expected attribute, being the trained panelists free to elect the attributes (descriptors) that they felt during the sample sensory evaluation. Finally, the overall sensory perceptions were graded using a similar continuous scale, being determined the complexity and the persistence sensations. For complexity, the panel evaluated the combination of the different positive sensations perceived for each olive oil. A high number of sensations perceived results in a great complexity. Contrary, a low number of sensations decrease the score of this parameter. In which concerns persistence, the panel ranked the feelings of the different sensations that persist in the mouth along the time. Long periods would mean a high persistence and if the sensations disappear a low persistence was scored.

\subsection{Fatty acids composition}

Fatty acids were determined following the European Community Regulation EEC/2568/91 from 11th July. A Chrompack CP 9001 chromatograph with a split-splitless injector, a FID detector and a Chrompack CP-9050 autosampler was used. Fatty acids separation was achieved using a Select FAME fused silica capillary coated column ( $50 \mathrm{~m} \times 0.25 \mathrm{~mm}$ i.d.) (Agilent). Helium was used as carrier gas (internal pressure equal to $110 \mathrm{kPa}$ ). The detector and injector temperatures were equal to $250{ }^{\circ} \mathrm{C}$ and $230{ }^{\circ} \mathrm{C}$, respectively. A split ratio of $1: 50$ was used being injected $1 \mu \mathrm{L}$. Fatty acids levels were expressed in relative percentage, determined by internal normalization of the chromatographic peak area eluting between myristic and lignoceric methyl esters. Peaks identification and quantification were carried out using a control (olive oil 47118, from Supelco) and a certified fatty acids methyl esters standard mixture (Sigma, Spain).

\subsection{Vitamin E content}

Vitamin $\mathrm{E}$ content was obtained by adding the individual tocopherol contents ( $\alpha$-, $\beta$ - and $\gamma$-), which were determined according to the ISO 9936 (2006), with some modifications (Rodrigues, Casal et al., 2018). Tocopherols standards and the internal standard 2-methyl-2-(4,8,12trimethyltridecyl)chroman-6-ol (tocol) were respectively from Sigma (Spain) and Matreya Inc. (USA). Fifty mg of filtered olive oil and tocol were dissolved in n-hexane and centrifuged at 13,000 rpm during $5 \mathrm{~min}$ being then the supernatant analyzed by HPLC. A liquid chromatograph comprised a data unit (Jasco, Japan), a Pump (PU-1580) and a fluorescence detector $(\lambda$ exc $=290 \mathrm{~nm} ; \lambda$ em $=330 \mathrm{~nm})$. The equipment and the analysis conditions are described in detail by Rodrigues, Casal et al. (2018).

\subsection{Total phenols content}

The total phenols contents were assessed using the methodology described by Capannesi, Palchetti, and Mascini (2000) with some modifications. Briefly, $2.5 \mathrm{~g}$ of olive oil were diluted with $2.5 \mathrm{~mL}$ of $\mathrm{n}$ hexane $(1: 1 \mathrm{w} / \mathrm{v})$ and extracted three times $(5 \mathrm{~min}$ of centrifugation at 
$5,000 \mathrm{rpm}$ ) with $2.5 \mathrm{~mL}$ of methanol/water (80:20; v/v). After $1 \mathrm{~mL}$ of the extract was added to $1 \mathrm{~mL}$ of Folin-Ciocalteau reagent, plus $1 \mathrm{~mL}$ of $\mathrm{Na}_{2} \mathrm{CO}_{3}$ solution $(7.5 \%)$ and $7 \mathrm{~mL}$ of purified water. The mixtures were then stored overnight, being the spectrophotometric analysis performed at $\lambda=765 \mathrm{~nm}$ using a calibration curve of caffeic acid in methanol $(0.04-0.18 \mathrm{mg} / \mathrm{mL})$. In accordance, the results were expressed as $\mathrm{mg}$ of caffeic acid equivalents per $\mathrm{kg}$ of olive oil (mg CAE $/ \mathrm{kg}$ ).

\subsection{Oxidative stability (Rancimat)}

The oxidative stability (OS) was established by measuring the oxidation induction time using a Rancimat 743 apparatus (Metrohm CH, Switzerland) following the methodology previous described by Rodrigues, Dias, Veloso, Pereira, and Peres (2016).

\subsection{Statistical analysis}

The possible effects of the tree cultivar and the crop year on the different physico-chemical and sensory parameters of olive oils were evaluated using one-way analysis of variance (one-way ANOVA), followed by the post-hoc multi-comparison Tukey's test if a significant statistical effect was found ( $P$-value $<0.050)$. The one-way ANOVA results were given in boxplots. The existence of bivariate correlations between fatty acids contents and sensory attributes of olive oils was evaluated based on the linear Pearson correlation coefficients $(R$ Pearson). The possible influences of olive tree cultivar (i.e., cvs. Lentisca, Madural, Rebolã, Redondal, Verdeal or Verdeal Transmontana) or crop years (2016 and 2017) on the chemical and sensory characteristics of olive oils extracted from olives of centenarian trees was further evaluated using principal component analysis (PCA) as an unsupervised pattern recognition tool. For minimizing data variability, data were centered and scaled. The statistical analysis was performed using the Subselect (Cadima, Cerdeira, \& Minhoto, 2004; Cadima, Cerdeira, Silva, \& Minhoto, 2012; Kuhn \& Johnson, 2013) and MASS (Venables \& Ripley, 2002) packages of the open source statistical program R (version 2.15.1).

\section{Results and discussions}

\subsection{Quality and physicochemical parameters}

In order to evaluate the quality of the varietal olive oils obtained from minor cultivars centenarian trees, different quality parameters were determined during two seasons (2016 and 2017), namely free acidity (FA), peroxide value (PV), extinction coefficients at 232 and $270 \mathrm{~nm}\left(\mathrm{~K}_{232}\right.$ and $\left.\mathrm{K}_{270}\right)$ and quality grade classification according to the sensory evaluation (Table 1 ). The obtained results show a consistently low FA without a clear cultivar pattern. In 2016 all samples were within a very narrow range, from $0.15 \%$ to $0.23 \%$, with $c v$. Redondal and $c v$. Verdeal Transmontana cultivars having the lowest FA and $c v$. Lentisca the highest. In 2017 the FA increased slightly, varying from $0.26 \%$ (cvs. Lentisca and Verdeal Transmontana) and $0.32 \%$ ( $c v$. Redondal). According to the results obtained for this parameter, it was verified that there is a low global free acidity (from 0.2 to $0.3 \%$ ), below the limit of 0.8\% established by Commission Delegated Regulation (EU) 2015/ 1830 of 8th July for the EVOO category. It was noticed that in the crop year of 2017 the results are globally higher than in 2016. This fact could be related with the extreme drought verified in 2017, during all season, that might have induced some increased hydrolysis in the fruit, but all values were generally low. For the peroxide value, indicative of oxidation, the lower values were also observed in 2016, ranging from 1.4 ( $c v$. Redondal) to $3.6 \mathrm{mEqO}_{2} / \mathrm{kg}$ olive oil ( $c v$. Madural) while in 2017, the values varied from 3.0 ( $c v$. Rebolã) and $5.2 \mathrm{mEqO}_{2} / \mathrm{kg}$ olive oil ( $c v$. Lentisca). Once again all PVs were below the $20 \mathrm{mEqO}_{2} / \mathrm{kg}$ maximum limit established by Commission Delegated Regulation (EU) 2015/1830 of 8th July for the classification of olive oil as EVOO. For

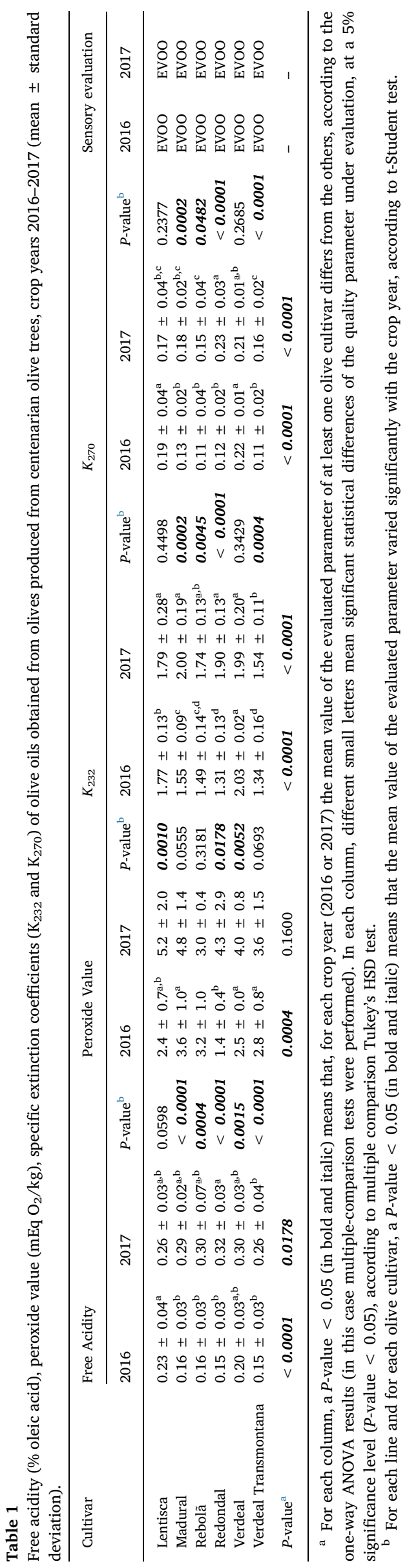


the extinction coefficients, namely $\mathrm{K}_{232}$, the values were lower than 2.03 in both years, with the lowest observed in $c v$. Redondal (2016, $1.31 \pm 0.13$ ), as opposed to $c v$. Verdeal that presented the highest one $(2016,2.03 \pm 0.02)$. For the $K_{270}$, the values varied from 0.11 (cvs. Rebolã and Verdeal Transmontana in 2016) and 0.23 (cv. Redondal, in 2017). All extinction coefficient values were within the legal limits established by the European Community Regulation EEC/2568/91 from 11th July for EVOO, with the exception of $c v$. Redondal in 2017, being therefore considered VOO. All olive oils were submitted to a sensory analysis, being all classified as EVOO without sensorial defects, and with a mean fruity note intensity above zero.

Although statistical differences were found for some quality parameters ( $P$-value $<0.0001$ ) between varieties (in the same crop year), and between years (within the same variety), the physicochemical levels were generally low, indicative of small hydrolysis and oxidation of the oils. This is not surprising since the olive oils were fresh and obtained from healthy fruits, harvested manually at an ideal ripening stage, transported to the laboratory in a short time and extracted on the same day, minimizing the risk of hydrolysis and oxidation of fatty acids. These results confirm that a correct handling of the fruits enables to produce high quality olive oil, with the genetic ground having a low influence on these parameters, in line with the results obtained by other authors (e.g.: Chiappetta, Muto, Muzzalupo, \& Muzzalupo, 2017; Xiang et al., 2017; Reboredo-Rodríguez, González-Barreiro, Cancho-Grande, Simal-Gándara, \& Trujillo, 2018). Table 2 details the oxidative stability (OS), total phenols (TP), vitamin E, and the oleic acid/linoleic acid ratio of the olive oils. Significant differences between cultivars ( $P$-value $<$ 0.0001 ) were observed in the OS, with the crop year having also a significant influence in this parameter for the cvs. Madural and Rebolã ( $P$-value $<0.0001)$. Consistently, the highest values, $32.6 \mathrm{~h}$ (2016) and $33.6 \mathrm{~h}$ (2017) were observed in the olive oils of $c v$. Redondal, and the lowest ones, $10.8 \mathrm{~h}$ (2016) and $14.7 \mathrm{~h}$ (2017) in $c v$. Madural. This parameter is of high importance once it could help to predict the shelf life of the olive oils and the capacity to prevent the oxidation process. According to the obtained results the following order could be established for shelf life: Redondal > Verdeal Transmontana > Verdeal > Lentisca > Rebolã > Madural. The global range of OS values obtained is similar to the ones obtained by other authors for different monovarietal olive oils considering analogous analytical conditions (e.g. Tura et al., 2007). And, for Verdeal Transmontana and Redondal, are in the same order of the values obtained for the two most common cultivars in Portugal (Galega Vulgar and Cobrançosa) (Peres et al., 2016). The TP content seemed to be significantly influenced by olive cultivar $(P$ value $<0.0001$ ), ranging from 75 to $135 \mathrm{mg} \mathrm{CAE} / \mathrm{kg}$ of olive oil in 2016 and from 164 to $363 \mathrm{mg}$ CAE/kg of olive oil in 2017, with the highest values consistently observed in $c v$. Redondal and the lowest ones for $c v$. Verdeal. The TP contents were also significantly influenced $(P$-value $<0.0001)$ by crop year with values consistently higher in 2017 (Table 2). This behavior was expected since the final amount of phenolic compounds, which are secondary plant metabolites, is influenced by diverse factors such as cultivar, geographical origin, agroclimatic conditions, degree of maturation, fruit freshness before extraction, extraction method and storage conditions (Ghisoni et al., 2019; Malheiro, Rodrigues, \& Pereira, 2015; Olmo-García et al., 2019; Wani et al., 2018). Due to their sensory characteristics and antioxidant activity, phenolic compounds are important for the sensory profile of the olive oils, contributing to olive oil resistance towards oxidation, while constituting interesting antioxidant sources for olive oil consumers. Still, within the antioxidants, the role of vitamin $\mathrm{E}$ is also well known on human health. According to Reboredo-Rodríguez et al. (2016), which characterized VOO produced with indigenous Galician varieties, antioxidants, namely the phenolic compounds, played an important role concerning the intensity and the release time of certain aromatic compounds, during the consumption of VOO, pointing out the relevance of the phenolic compounds at the sensory perception level. The results (Table 2) demonstrated that the amounts of vitamin E were

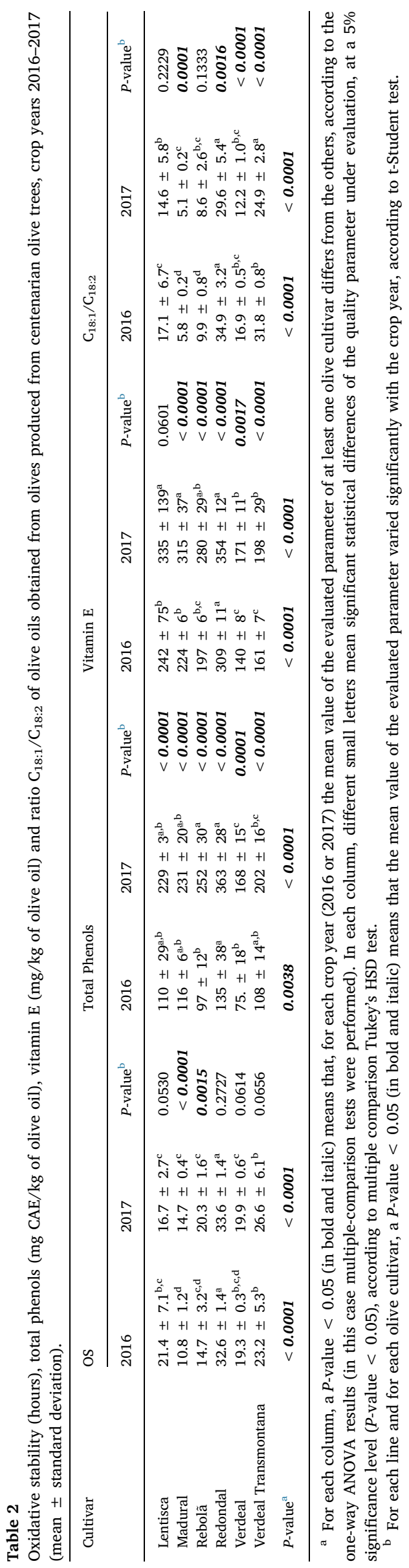


dependent of the studied factors, the olive cultivar and crop year $(P$ value $<0.0001$ ). Again, $c v$. Redondal was richest in vitamin $\mathrm{E}$, with $309 \mathrm{mg} / \mathrm{kg}$ in 2016, and $354 \mathrm{mg} / \mathrm{kg}$ in 2017, while $\mathrm{cv}$. Verdeal was the poorest, with $140 \mathrm{mg} / \mathrm{kg}$ in 2016 and $172 \mathrm{mg} / \mathrm{kg}$ in 2017. These results agree in general with the ones obtained for different cultivars in different parts of the world, with average amounts varying from 50 to $500 \mathrm{mg} / \mathrm{kg}$ of olive oil, and also for the Portuguese cultivars (Galega Vulgar and Cobrançosa) (Peres et al., 2016) suggesting that the amount of vitamin E highly depends on the olive cultivar (Beltrán et al., 2010; Reboredo-Rodríguez et al., 2016; Tura et al., 2007) but also influenced by other factors like maturation process and agro-climatic conditions. In the present work, the severe drought observed during the year 2017 (data not shown) probably promoted the synthesis of these antioxidants, perhaps as a protection of the plant against the increased environmental stress (Beltrán et al., 2005).

The fatty acids composition is a genuineness parameter for olive oils according to the Commission Delegated Regulation (EU) 2015/1830 of 8th July and can also be used for cultivar discrimination (Kritioti et al., 2018), while the unsaturation degree settles the oxidative susceptibility, and consequently the shelf life of olive oils. The oleic/linoleic acid ratios of the olive oil samples were statistical influenced $(P$ value $<0.0001$ ) by the olive cultivar and in some cases by the crop year (Table 2). In both years, Madural was the cultivar that presented the lowest ratios, with values of 5.75 and 5.11 respectively for 2016 and 2017. On opposition, the highest ratios were obtained for $c v$. Redondal, with 34.9 and 29.6 for 2016 and 2017, respectively. Peres et al. (2016) obtained values in the order of 17.8 and 8.3, respectively for the Galega Vulgar and Cobrançosa cultivars. In general, the $\mathrm{C}_{18: 1} / \mathrm{C}_{18: 2}$ ratio followed the order Redondal > Verdeal Transmontana > Verdeal ${ }^{>}$Lentisca > Rebolã > Madural, highly consistent with the results observed for the OS. It could also be observed that the ratios obtained in 2017 were lower than those of 2016, for all cultivars. This could be indicative that under high summer temperatures and drought (data not shown), as the ones observed in 2017, the fatty acids biosynthesis pathway is altered, probably in favour of linoleic acid. According to Boskou, Blekas, and Tsimidou (2006), genetics, climate and agronomic conditions influence the diversity of fatty acids.

If all the information of the Table 2 is analysed together, it could be inferred that $c v$. Redondal is simultaneously the cultivar with the higher amount in antioxidants and also higher $\mathrm{C}_{18: 1} / \mathrm{C}_{18: 2}$ ratios, a combined situation that seems to be determinant for an increased OS. Conjugating both OS and the $\mathrm{C}_{18: 1} / \mathrm{C}_{18: 2}$ (mean values per cultivar for both years), a significant positive linear correlation was obtained $(R$-Pearson $=$ +0.9704 ; $P$-value $=0.0013$ ), pointing out that the OS of olive oils is promoted by higher $\mathrm{C}_{18: 1} / \mathrm{C}_{18: 2}$ ratios.

\subsection{Descriptive sensory profile}

The sensory profiles of the olive oils obtained from centenarian trees of minor cultivars Lentisca, Madural, Rebolã, Redondal, Verdeal and Verdeal Transmontana are shown in Figs. 1, 2 and 3. In those figures, an average of both crop years (2016-2017) of each attribute were presented once the global aim was to define the sensory profile of each cultivar. Fig. 1 showed the olfactory profile of the different olive oils, which note intensity perception is graded from 0 to 10 . All olive oils presented a fruity intensity higher than 0 without any sensory defect, allowing their classification as EVOO (Commission Delegated Regulation (EU) 2015/1830 of 8th July). In all samples the fruity olfactory sensation was "green" and no statistical differences were found regarding this parameter, which intensities varied from 5.0 in the $c v s$. Rebolã and Verdeal to 5.9 in $c v$. Madural. Still, different fruits sensations were found (Fig. 1). All oils presented a tomato note sensation, with a broad range of perceived intensities that varied from 1.0 to 7.5. Although statistically similar for all cultivars, the highest mean values for this attribute were observed in $c v$. Verdeal Transmontana (4.2) and the lowest in $c v$. Verdeal (3.0). Low intensities were observed for "apple" aroma and two statistical different groups could be found ( $P$ value $\leq 0.05$ ): higher intensities were attributed to the group constituted by the cvs. Madural, Rebolã, Redondal and Verdeal Transmontana, with averages around 3.8, and lower to the group constituted by $c v s$. Lentisca (3.1) and Verdeal (2.7). For the "banana" aroma, two statistical different groups were also obtained $(P$-value $\leq 0.05)$ (Fig. 1), being this aroma characteristic of $c v$. Madural and Rebolã, and absent in Redondal and Verdeal olive oils. All oils presented "dry fruits sensation" with similar scores, between 1.6 ( $c v$. Verdeal) and 2.4 ( $c v$. Redondal). In previous works "dry fruits note" is an attribute commonly found in the olive oils produced in the geographical area (Rodrigues et al., 2019) and so, it could be considered a typical note that characterizes the "terroir" of the region. Rodrigues et al. (2019) considered dry fruits as a standard sensory attribute of olive oils from different cultivars, which was perceived in all olive oils evaluated. In the present work other fruitbased sensations were also perceived by the panelists, including tomato, apple, banana, fresh herbs, cabbage and tomato leaves, which were classified as positive attributes. However, since their perceptions were residual and for a scarce number of samples, they were not statistically evaluated. Green cherry was noticeable in cvs. Lentisca and Madural with mean values of 1.6 and 0.4 , respectively, while apricot sensation appeared in cvs. Lentisca (1.7), Madural (0.5) and Verdeal Transmontana (0.1). The kiwi notes were only perceptible in $c v$. Madural (0.4). Lukić et al. (2017) and Lukić et al. (2018) when studying monovarietal olive oils of cvs. Oblica Buža and Istarska bjelica, reported sensory attributes like apple, tomato, almond, aromatic herbs and chicory/rocket, which perceptions differ according to the cultivar.

Concerning herbaceous sensations, attributes of fresh herbs, tomato leaves and cabbage (sensation of cut cabbage) were found. Within these attributes, fresh herbs were generally the dominant notes but statistically different between cultivars $(P$-value $\leq 0.05)$. The olive oils from $c v$. Verdeal Transmontana presented the highest mean value (4.0) and the $c v$. Verdeal the lowest one (2.5). Similarly, cut cabbage sensation was significantly $(P$-value $\leq 0.05$ ) higher for $c v$. Verdeal Transmontana (3.8) and Rebolã (3.5) oils and lower for $c v$. Lentisca (0.4), with middle values for the remaining cultivars. For the "tomato leaves" attribute Verdeal Transmontana presented the highest values (2.6) while cvs. Lentisca and Rebolã (1.6) scored the lowest (Fig. 1). The obtained results for herbaceous notes are the main responsible for the green fruity aroma of the olive oils. Fig. 2A shows the values for fruity intensity and for the basic sweet, bitter and pungent attributes. All the analyzed olive oils showed similar fruity intensity, with mean values from 5.1 ( $c v$. Rebolã) to 5.8 ( $c v$. Verdeal Transmontana) (Fig. 2A). Concerning the basic gustatory attributes there were statistically differences $(P$ value $<0.05)$ in sweet and pungent notes. Olive oils from cvs. Redondal (1.6), Verdeal (1.6) and Verdeal Transmontana (1.5) were significantly less sweet than Madural (3.3). For pungent attribute two distinct significantly ( $P$-value $\leq 0.05$ ) groups were found, one with higher values constituted by $c v s$. Lentisca, Madural, Verdeal and Verdeal Transmontana, and other with lower values formed by $c v$. Rebolã and Redondal. Despite the diversity of values observed for the bitter attribute, no significant differences were observed. Different studies demonstrated that bitter and pungent sensory attributes are correlated with different groups of phenolic compounds (Genovese et al., 2018). Nevertheless in our work, only total phenols were evaluated. Similar values of basic flavors were observed by Reboredo-Rodríguez et al. (2016) and Reboredo-Rodríguez et al. (2018) in olive oils from autochthonous olive cultivars from Galicia (Spain). García-Mesa, PereiraCaro, Fernández-Hernández, Civantos, and Mateos (2008) referred that basic sensations may vary according to the lipid composition, with matrices richer in monounsaturated fatty acids (MUFA) being more bitter and pungent than matrices rich in polyunsaturated acids (PUFA). However, our results did not follow this same tendency and no significant correlations were found between MUFA and bitter and pungent attributes. Nevertheless, a significantly negative correlation $(R$ Pearson $=-0.8448 ; \quad P$-value $=0.0342)$ was determined between 

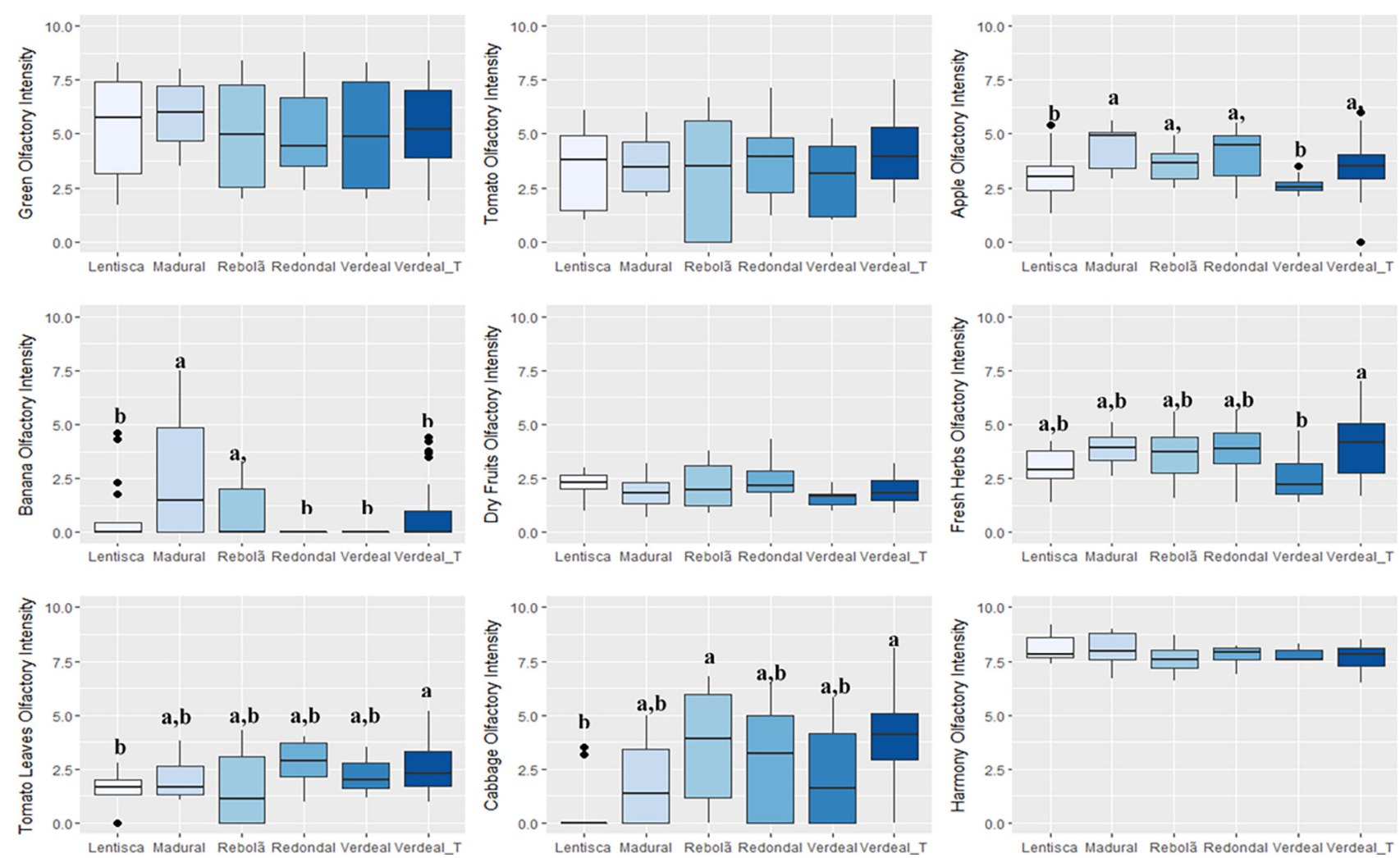

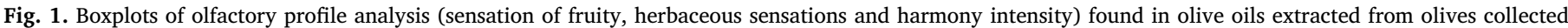

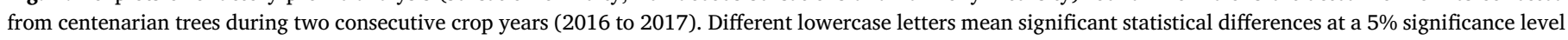
(one-way ANOVA followed by the Tukey's multi-comparison test).

MUFA and the intensity of the sweet sensation. This attribute was also significantly positive correlated $(R$-Pearson $=\quad+0.9391 ; \quad P$ value $=0.0054$ ) with PUFA and bitterness also showed a positive correlation with the amount of saturated fatty acids $(R$-Pearson $=$ $+0.8252 ; P$-value $=0.0432$ ). Gustatory attributes included fruity sensations of tomato, apple, banana and dry fruits (Fig. 2B). Tomato sensation was the most pronounced, with mean values from 1.0 to 7.6 , but no significant differences were observed between cultivars. The same trends were observed for apple and dry nut sensations, similar in all cultivars. These attributes were well perceptible by the panelists in all olive oils, but no statistical differences were observed between cultivars, which could be attributed to the "terroir" of the region since they were also reported in previous works with other cultivars (Rodrigues, Oliveira et al., 2018). A contrary situation was observed for banana gustatory attribute, with two significantly different groups observed $(P$ value $\leq 0.05$ ), being significant amounts detected in cvs. Madural (3.1) and Lentisca (3.0), whereas for Verdeal (1.0) and Verdeal Transmontana (0.9) the attribute is practically absent (Fig. 2B), and so, could be foreseen as potential markers of the former cultivars. As in olfactory sensations, other fruit gustatory attributes, like green cherry, apricot and kiwi were also found, but only perceived in olive oils of some cultivars. Cherry gustatory attribute was only noticeable in $c v s$. Lentisca (1.9) and Madural (0.3); apricot sensation appeared in $c v s$. Lentisca (2.2), Madural (0.5) and Verdeal Transmontana (0.1); and, kiwi notes were noticeable in $c v s$. Madural (0.4), Lentisca (0.3), Rebolã (0.8) and Verdeal Transmontana (0.2). Herbaceous sensations were dominated by notes of fresh herbs, tomato leaves and cabbage (Fig. 2A). The values of fresh herbs and tomato leaves were similar in all the cultivars. For cabbage sensation, significant differences $(P$-value $\leq 0.05)$ were observed between cultivars, being higher values obtained for Verdeal Transmontana and Redondal, whilst this attribute was absent for Lentisca olive oils. Some authors (Caporale, Policastro, \& Monteleone, 2004) reported that the sensation of fresh herbs can significantly increase the perception of bitterness. Nevertheless, it was not observed in the present work, being probably cultivar dependent. Harmony is defined as an overall sensation that's combines all the obtained perceptions by the taster: its classification is indicative of the equilibrium between the perceptions found, being usually attributed higher scores when different sensations are perceived but without high dominance of any. Two statistical different $(P$-value $\leq 0.05)$ groups were constituted: $c v$. Madural olive oils obtained the highest score whereas Rebolã and Verdeal Transmontana obtained the lowest (Fig. 2B).

At the level of gustatory-retronasal attributes cvs. Lentisca and Madural were significantly more complex than the oils from $c v$. Rebolã, which was the less complex. Verdeal Transmontana originated more persistent olive oils whilst cvs. Rebolã and Redondal showed the lower persistence values. Also, Dias, Rodrigues, Veloso, Pereira, and Peres (2016) conclude that olive oil persistence was influenced by cultivar. Furthermore, Gerhardt et al. (2019) pointed out the importance of performing sensory analysis of VOO together with other techniques for establishing the exact commercial category of an olive oil, showing the need of the former analysis as a key complementary quality evaluation tool. However, since a variability of the sensory scores was observed for VOO samples assessed by different sensory test panels, the correct classification of the oil samples would require the fusion of the sensory data with the volatile profiles (VOC) and the use of multivariate supervised and/or unsupervised chemometric tools (e.g., PCA, LDA, $\mathrm{KNN}$ ). In the present study, it was also observed a high variability of the sensory scores attributed by different trained panelists during the evaluation of some olive oil samples. Nevertheless, since the sensory profile was used to characterize olive oils obtained from minor cultivars, the found variability does not constitute a commercial problem. On the other hand, the assessment sensory variability reported may be tentatively attributed to the fact that sensory panels are trained to detect and score the defects at the fruity intensity levels and less focused on the evaluation of the positive attributes, increasing therefore the scores 
A
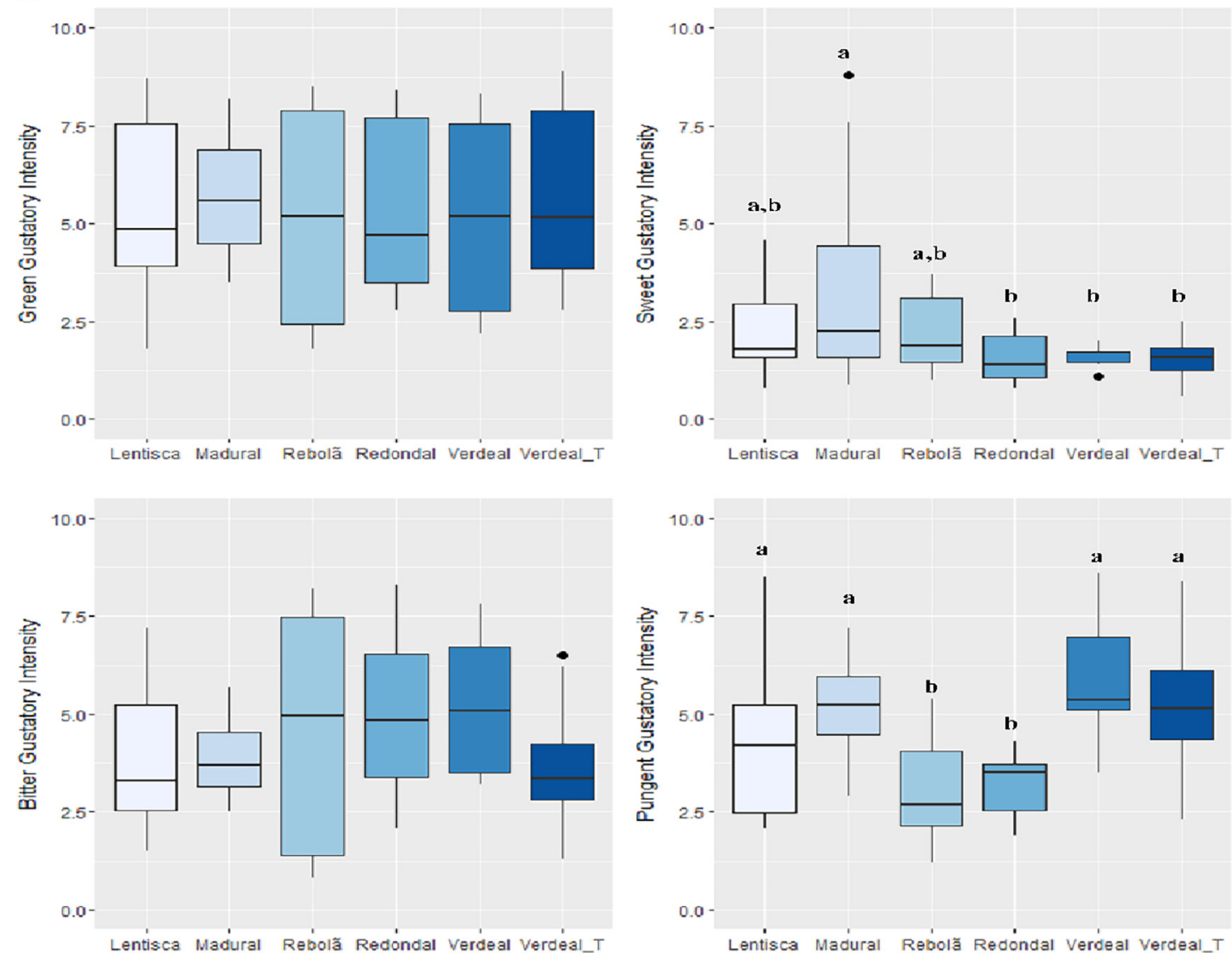

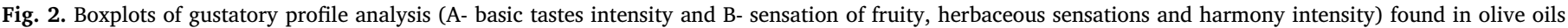

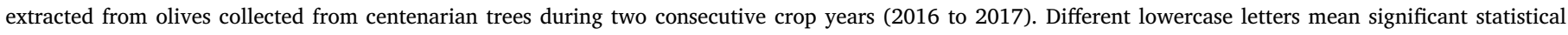
differences at a 5\% significance level (one-way ANOVA followed by the Tukey's multi-comparison test).

variability.

\subsection{Merging quality, physicochemical and sensory attributes towards the} simultaneous discrimination of olive oil by crop year and olive cultivar

The previous discussed effects of the crop year and/or olive cultivar on the quality, physicochemical and perceived intensities of positive sensory attributes, pointed out the opportunity of simultaneously discriminating the olive oils produced from the centenarian trees according to the referred two factors by merging all the information collected, using a low-level fusion strategy. For that, a PCA was performed and, as can be inferred from Fig. 4, the fused data allowed: (i) first, discriminating olive oils according to the crop year, accordingly, olive oils produced on 2016 were located on the negative region of the 1st principal component, PC1, mainly due to the contribution of $\Delta K$, $\mathrm{C}_{18: 1} / \mathrm{C}_{18: 2}$ and the sweet gustatory sensation, and those produced on 2017, located on the positive region of PC1, mainly due to the levels of free acidity, total phenols, olfactory and gustatory green and tomato sensations, and the bitter intensity attribute; and (ii) secondly, for each year, olive oils were differentiated by olive cultivar, based on PC2 and PC3, due to the miscellaneous contribution of the assessed physicochemical and sensory parameters. For each crop year the space location of the olive oils belonging to the same olive cultivar substantially differ, with the exception of $c v s$. Verdeal Transmontana and Lentisca, possibly indicating that olive oils produced from the two latter cultivars were less prone to the known influence of climatic conditions. Globally, these findings are in agreement with the detailed study described in the previous sections concerning quality, physicochemical and sensory data, which pointed out the significant influences of both crop year and olive cultivar on the overall composition and sensory profile of olive oils. In the present work, PCA was applied to evaluate the possibility of differentiating the olive oils according to the olive variety and crop year. The use of statistical tools for authentication purposes is a hot research topic in which concerns olive oils' chemical and sensory characterization. Independently of the performed analysis, taking into account the type of the samples studied and the aims of the work, the finding herein reported should be observed and discussed using a holistic approach. In fact, the chemical and sensory diversity of olive oils is greatly influenced by many different factors such as genotype, agronomic and ecophysiological conditions, ripening process and postharvest treatments, as well as the extraction and storage conditions of the olive oils. Also, sensory analysis is intrinsically dependent on the experience of the sensory experts, which that significantly contribute to the final sensory quality grading of the olive oil (Ghisoni et al., 2019). So, supervised multivariate analysis using different statistical tools, were recently successfully implemented, based on a metabolomics approach, to identify the phenolic and sterols compounds that correctly allow discriminating EVOOs from different cultivars grown in the same conditions (Valli et al., 2019). Similarly, Olmo-García et al. (2019) demonstrated the potential of using multi-class GC-MS data of minor composition fractions to correctly discriminate varietal olive oils from eight cultivars produced in the same conditions.

\section{Conclusions}

This work intended to characterize six minor olive cultivars produced from centenarian trees, aiming to contribute for their characterization, conservation and valorization as a way to preserve these 

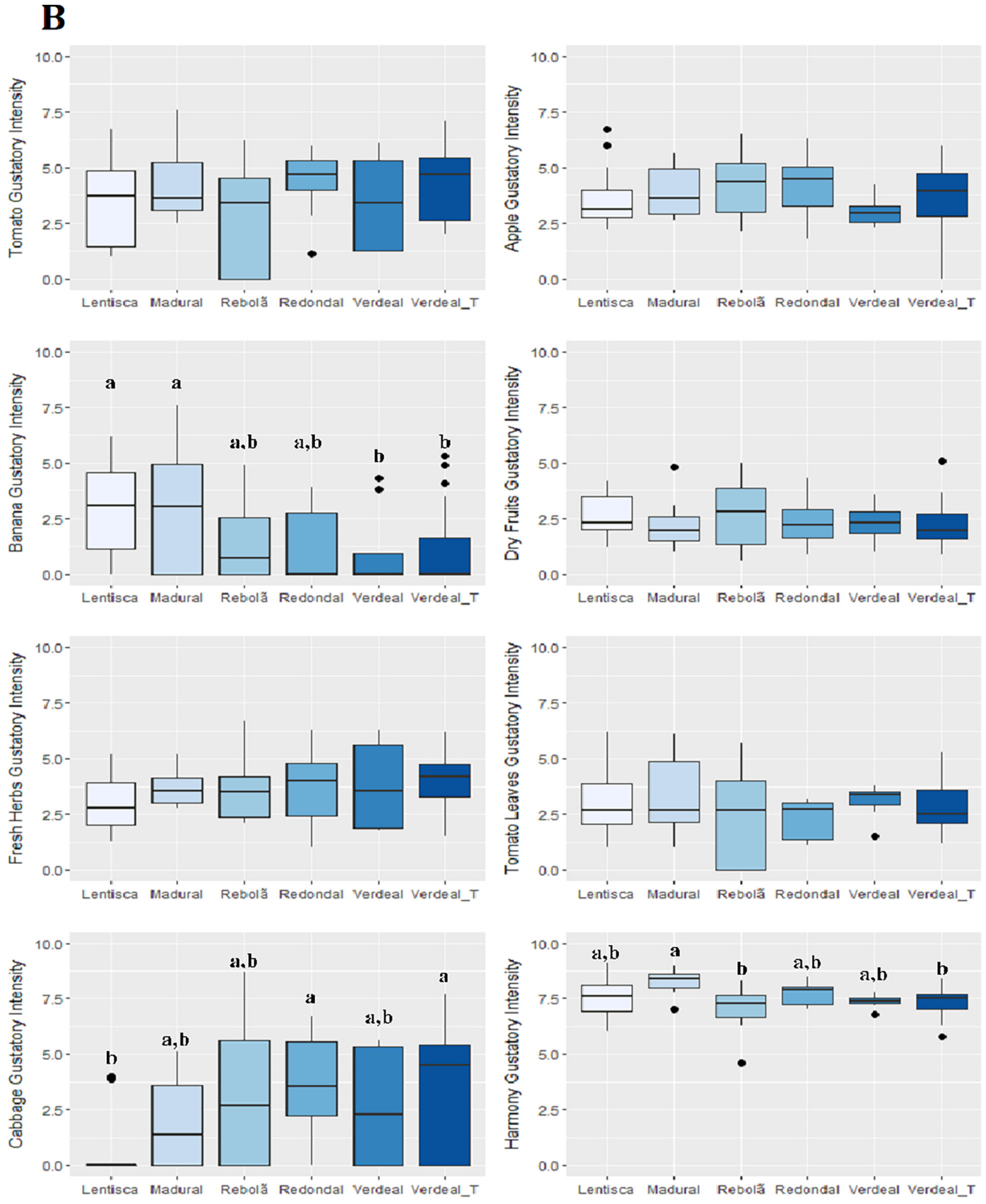

Fig. 2. (continued)

cultivars as part of the genetic identity of the Trás-os-Montes region. Of the 20 specimens of centenary trees selected for this study, it was possible to identify specimens that gave olive oils of exceptional quality. From the point of view of the quality, all fulfilled the requirements to be classified as EVOO, showing the importance of the field and technological work. At the chemical level the cultivar effect was visible, with $c v$. Redondal having higher resistance to oxidation, higher total phenol content, total tocopherols and oleic/linoleic acid ratios. For the organoleptic point of view some of the obtained oils showed an exquisite profile, with rare notes like green cherry (Lentisca and Madural), apricot (Lentisca, Madural and Verdeal Transmontana) and kiwi (Lentisca, Madural, Rebolã and Verdeal Transmontana). These selected trees are potential candidates to obtain differentiated oils while promoting the future preservation of the region genetic heritage. Furthermore, linear correlations were found between sweet and bitter sensations and the mean levels of monounsaturated, polyunsaturated 

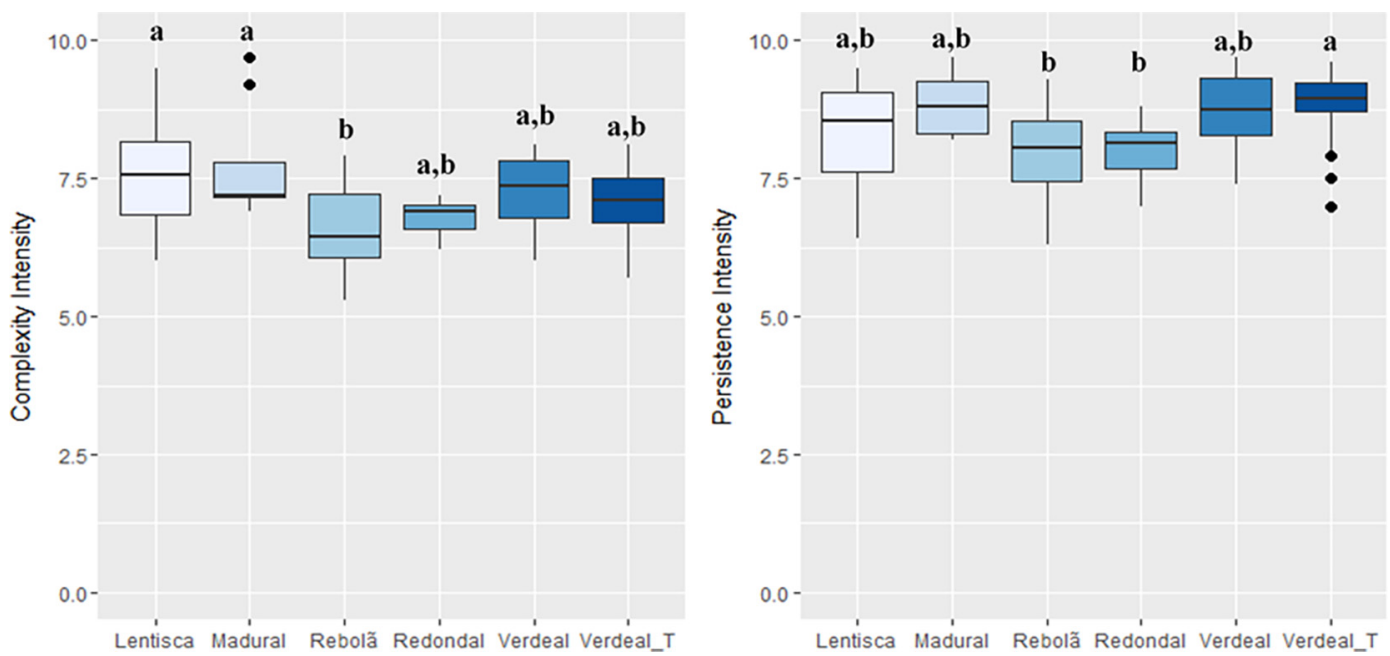

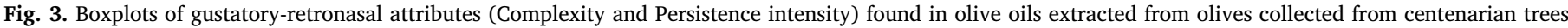

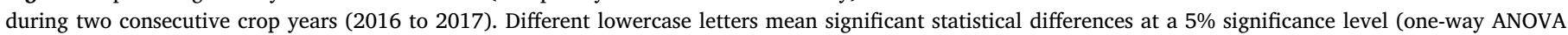
followed by the Tukey's multi-comparison test).

and/or saturated fatty acids, showing the influence of fatty acids on the olive oil organoleptic sensations. Finally, by merging the quality, physicochemical and sensory data allowed establishing a classification model that enabled assessing the olive oil according to the crop year as well as to identify the olive cultivar of each oil evaluated.

\section{Declaration of Competing Interest}

The authors declare that they have no known competing financial interests or personal relationships that could have appeared to influence the work reported in this paper.

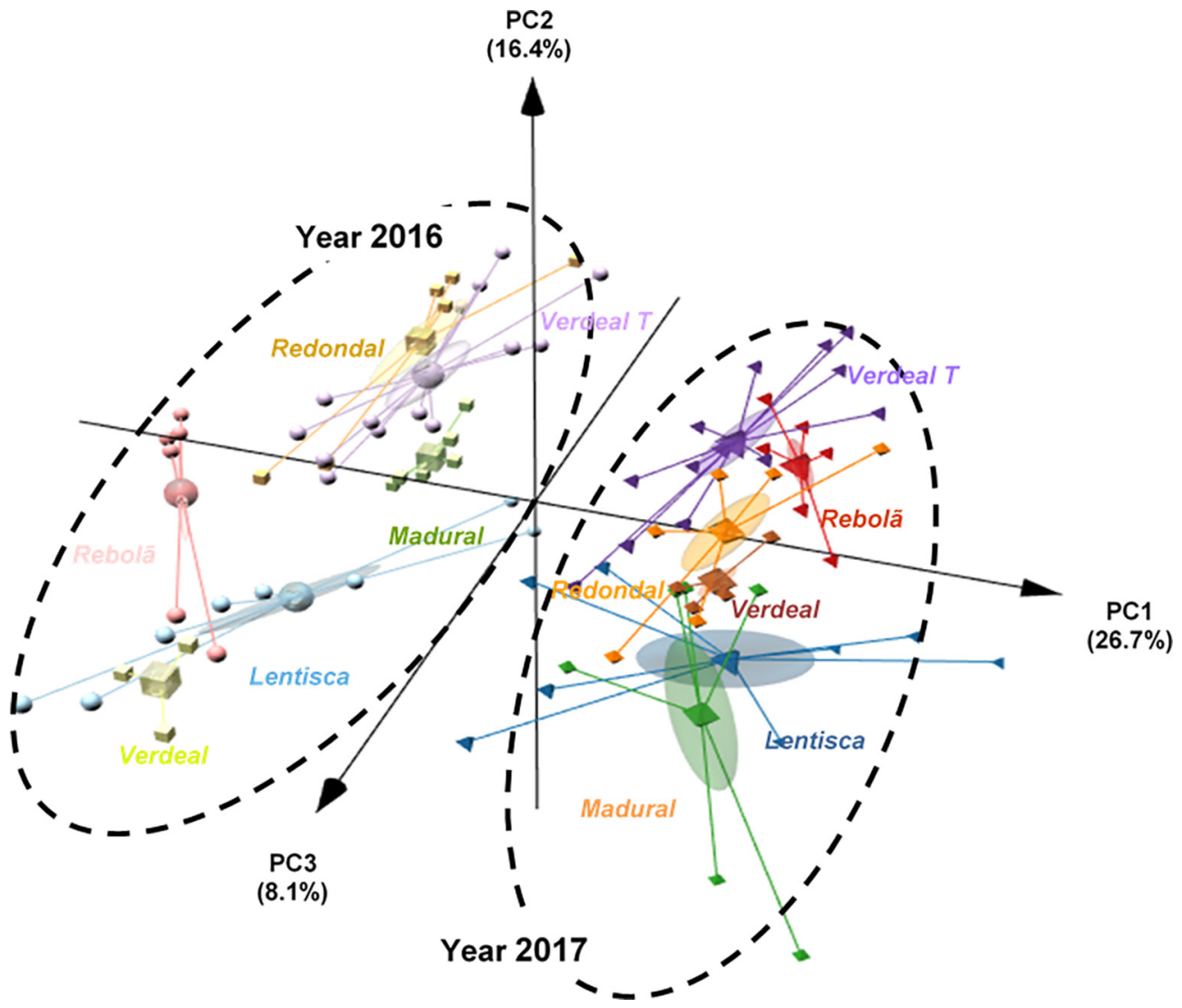

\section{Acknowledgements}

This work was financially supported by Strategic Project PEst-OE/ AGR/UI0690/2019 (CIMO), Project UID/QUI/50006/2013 (REQUIMTE-LAQV); and Project POCI-01-0145-FEDER-006984 (Associate Laboratory LSRE-LCM); all funded by Fundo Europeu de Desenvolvimento Regional (FEDER) through COMPETE2020-Programa Operacional Competitividade e Internacionalização (POCI) and by national funds through Fundação para a Ciência e a Tecnologia (FCT), Portugal. This work was also supported by PRODER project OliveOld Identificação e caraterização de oliveiras centenárias para obtenção de
Fig. 4. Principal component analysis (PC1: 26.7\%, PC2: $16.4 \%$ and PC3: $8.1 \%$ ): 3D plot showing the unsupervised pattern recognition according to olive cultivar (cvs. Lentisca, Madural, Rebolã, Redondal, Verdeal and Verdeal Transmontana) based on the quality and physicochemical parameters and sensory analysis profile contents found in olive oils obtained from olives collected from centenarian trees during two consecutive crop years (2016 to 2017). 
produtos diferenciados" n ${ }^{\circ}$ 53988. Nuno Rodrigues thanks FCT, POPHQREN and FSE for the Ph.D. Grant (SFRH/BD/104038/2014).

\section{Appendix A. Supplementary material}

Supplementary data to this article can be found online at https:// doi.org/10.1016/j.foodres.2019.108759.

\section{References}

Aparicio-Ruiz, R., García-González, D. L., Morales, M. T., Lobo-Prieto, A., \& Romero, I. (2018). Comparison of two analytical methods validated for the determination of volatile compounds in virgin olive oil: GC-FID vs GC-MS. Talanta, 187, 133-141.

Bajoub, A., Hurtado-Fernández, E., Ajal, E. A., Fernández-Gutiérrez, A., CarrascoPancorbo, A., \& Ouazzani, N. (2015). Quality and chemical profiles of monovarietal north Moroccan olive oils from "picholine Marocaine" cultivar: Registration database development and geographical discrimination. Food Chemistry, 179, 127-136.

Beltrán, G., Aguilera, M. P., Del Rio, C., Sánchez, S., \& Martínez, L. (2005). Influence of fruit ripening process on the natural antioxidant content of Hojiblanca virgin olive oils. Food Chemistry, 89, 207-215.

Beltrán, G., Jiménez, A., Del Rio, C., Sánchez, S., Martínez, L., Uceda, M., et al. (2010). Variability of vitamin $\mathrm{E}$ in virgin olive oil by agronomical and genetic factors. Journal of Food Composition and Analysis, 23, 633-639.

Blasi, F., Rocchetti, G., Montesano, D., Lucini, L., Chiodelli, G., Ghisoni, S., et al. (2018). Changes in extra-virgin olive oil added with Lycium barbarum L. carotenoids during frying: Chemical analyses and metabolomic approach. Food Research International, 105, 507-516.

Boskou, D., Blekas, G., Tsimidou, M. (2006). Olive oil composition. D. Boskou (Ed.), Olive oil, chemistry and technology (pp. 41-72). Champaign Illinois: AOCS Press. ISBN: 9780128043547.

Cadima, J., Cerdeira, J. O., \& Minhoto, M. (2004). Computational aspects of algorithms for variable selection in the context of principal components. Computational Statistics \& Data Analysis, 47, 225-236.

Cadima, J., Cerdeira, J. O., Silva, P. D., \& Minhoto, M. (2012). The subselect R package. $<$ http://cran.rproject.org/web/packages/subselect/vignettes/subselect. pdf $>$ Accessed 15/02/2016.

Capannesi, C., Palchetti, I., \& Mascini, M. (2000). Electrochemical sensor and biosensor for polyphenols detection in olive oils. Food Chemistry, 71, 553-562.

Caporale, G., Policastro, S., \& Monteleone, E. (2004). Bitterness enhancement induced by cut grass odorant (cis-3-hexen-1-ol) in a model olive oil. Food Quality and Preference, $15,219-227$.

Chiappetta, A., Muto, A., Muzzalupo, R., \& Muzzalupo, I. (2017). New rapid procedure for genetic characterization of Italian wild olive (Olea europaea) and traceability of virgin olive oils by means of SSR markers. Scientia Horticulturae, 226, 42-49.

Comission Delegated Regulation (EU) 2015/1830 of 8th July 2015: Amending Regulation (EEC) No 2568/91 on the characteristics of olive oil and olive-residue oil and on the relevant methods of analysis. Official Journal of the European Union L266, 9-13.

Del Monaco, G., Officioso, A., D’Angelo, S., La Cara, F., Ionata, E., Marcolongo, L., et al. (2015). Characterization of extra virgin olive oils produced with typical Italian varieties by their phenolic profile. Food Chemistry, 184, 220-228.

Dias, L. G., Rodrigues, N., Veloso, A. C. A., Pereira, J. A., \& Peres, A. M. (2016). Monovarietal extra-virgin olive oil classification: A fusion of human sensory attributes and an electronic tongue. European Food Research and Technology, 242, 259-270.

Fernandes, G. D., Ellis, A. C., Gámbaro, A., \& Barrera-Arellano, D. (2018). Sensory evaluation of high-quality virgin olive oil: Panel analysis versus consumer perception. Current Opinion in Food Science, 21, 66-71.

Garcia, B., Magalhães, J., Fregapane, G., Salvador, M. D., \& Paiva-Martins, F. (2012) Potential of selected Portuguese cultivars for the production of high quality monovarietal virgin olive oil. European Journal of Lipid Science and Technology, 114, 1070-1082.

García-Mesa, J. A., Pereira-Caro, G., Fernández-Hernández, A., Civantos, C. G. O., \& Mateos, R. (2008). Influence of lipid matrix in the bitterness perception of virgin olive oil. Food Quality and Preference, 19, 421-430.

García-Vico, L., García-Rodríguez, R., Sanz, C., \& Pérez, A. G. (2017). Biochemical aspects of olive freezing-damage: Impact on the phenolic and volatile profiles of virgin olive oil. LWT- Food Science and Technology, 86, 240-246.

Genovese, A., Yang, N., Linforth, R., Sacchi, R., \& Fisk, I. (2018). The role of phenolic compounds on olive oil aroma release. Food Research International, 112, 319-327.

Ghisoni, S., Lucini, L., Angilletta, F., Rocchetti, G., Farinell, D., Tombesi, S., et al. (2019). Discrimination of extra-virgin-olive oils from different cultivars and geographical origins by untargeted metabolomics. Food Research International, 121, 746-753.

Gerhardt, N., Schwolow, S., Rohn, S., Pérz-Cacho, P. R., Galán-Soldevilla, H., Arce, L., et al. (2019). Quality assessment of olive oils based on temperature-ramped HS-GCIMS and sensory evaluation: Comparison of different processing approaches by LDA, kNN, and SVM. Food Chemistry, 278, 720-728.

IOC, International Olive Council, Sensory Analysis of Olive Oil - Method for the
Organoleptic Assessment of Virgin Olive Oil applying to use a designation of origin, COI/T.20/Doc. no. 22 November 2005, 29pp.

ISO 9936, 2006. Animal and vegetable fats and oils - determination of tocopherol and tocotrienol contents by high-performance liquid chromatography.

Köseoğlu, O., Sevim, D., \& Kadiroğlu, P. (2016). Quality characteristics and antioxidant properties of Turkish monovarietal olive oils regarding stages of olive ripening. Food Chemistry, 212, 628-634.

Krichene, D., Allalout, A., Baccouri, B., Salvador, M. D., Fregapane, G., \& Zarrouket, M. (2010). Fatty acids, volatiles, sterols and triterpenic alcohols of six monovarietal Tunisian virgin olive oils. European Journal of Lipid Science and Technology, 112, 400-409.

Kritioti, A., Menexes, G., \& Drouza, C. (2018). Chemometric characterization of virgin olive oils of the two major Cypriot cultivars based on their fatty acid composition. Food Research International, 103, 426-437.

Kuhn, M., \& Johnson, K. (2013). Applied predictive modeling. New York: Springer Science Business Media.

Lukić, I., Horvat, I., Godena, S., Krapac, M., Lukić, M., Vrhovsek, U., et al. (2018). Towards understanding the varietal typicity of virgin olive oil by correlating sensory and compositional analysis data: A case study. Food Research International, 112, 78-89.

Lukić, I., Žanetić, M., Špikac, M. J., Lukić, M., Koprivnjak, O., \& Bubola, K. B. (2017). Complex interactive effects of ripening degree, malaxation duration and temperature on Oblica $c v$. virgin olive oil phenols, volatiles and sensory quality. Food Chemistry, 232, 610-620.

Malheiro, R., Rodrigues, N., Pereira, J. A. (2015). Olive oil phenolic composition as affected by geographic origin, olive cultivar and cultivation systems. In "Olive and Olive Oil Bioactive Constituents" Ed. Dimitrios Boskou. Cap. 4, AOCS Press., 93-121. ISBN 978-1-630670-41-2. 30p.

Olmo-García, L., Polari, J. J., Li, X., Bajoub, A., Fernández-Gutiérrez, A., Wangb, S. C., et al. (2019). Study of the minor fraction of virgin olive oil by a multi-class GC-MS approach: Comprehensive quantitative characterization and varietal discrimination potential. Food Research International, 125, 108649.

Peres, F., Martins, L. L., Mourato, M., Vitorino, C., Antunes, P., \& Ferreira-Dias, S. (2016). Phenolic compounds of "Galega Vulgar" and "Cobrançosa" olive oils along early ripening stages. Food Chemistry, 211, 51-58.

Reboredo-Rodríguez, P., González-Barreiro, C., Cancho-Grande, B., Valli, E., Bendini, A., Toschi, T. G., et al. (2016). Characterization of virgin olive oils produced with autochthonous Galician varieties. Food Chemistry, 212, 162-171.

Reboredo-Rodríguez, P., González-Barreiro, C., Cancho-Grande, B., Fregapane, G., Salvador, M. D., \& Simal-Gándara, J. (2015). Blending Local olive oils with Arbequina or Picual oils produces high quality, distinctive EVOOs. European Journal of Lipid Science and Technology, 117, 1238-1247.

Reboredo-Rodríguez, P., González-Barreiro, C., Cancho-Grande, B., Simal-Gándara, J., \& Trujillo, I. (2018). Genotypic and phenotypic identification of olive cultivars from north-western Spain and characterization of their extra virgin olive oils in terms of fatty acid composition and minor compounds. Scientia Horticulturae, 232, 269-279.

Rodrigues, N., Casal, S., Peres, A. M., Baptista, P., Bento, A., Martín, H., et al. (2018). Effect of olive trees density on the quality and composition of olive oil from $\mathrm{cv}$. Arbequina. Scientia Horticulturae, 238(19), 222-233.

Rodrigues, N., Dias, L. G., Veloso, A. C. A., Pereira, J. A., \& Peres, A. M. (2016). Monitoring olive oils quality and oxidative resistance during storage using an electronic tongue. LWT - Food Science and Technology, 73, 683-692.

Rodrigues, N., Marx, I., Casal, S., Dias, L. G., Veloso, A. C. A., Pereira, J. A., et al. (2019). Application of an electronic tongue as a single-run tool for olive oils'physicochemical and sensory simultaneous assessment. Talanta, 197, 363-373.

Rodrigues, N., Oliveira, L., Mendanha, L., Sebti, M., Dias, L. G., Oueslati, S., et al. (2018). Olive oil quality and sensory changes during house-use simulation and temporal assessment using an electronic tongue. Journal of the American Oil Chemists' Society, 95, 1121-1137.

Salimonti, A., Simeone, V., Cesari, G., Lamaj, F., Cattivelli, L., Perri, E., et al. (2013). A first molecular investigation of monumental olive trees in Apulia region. Scientia Horticulturae, 162, 204-212.

Tura, D., Gigliotti, C., Pedò, S., Failla, O., Bassi, D., \& Serraiocco, A. (2007). Influence of cultivar and site of cultivation on levels of lipophilic and hydrophilic antioxidants in virgin olive oils (Olea europea L.) and correlations with oxidative stability. Scientia Horticulturae, 112, 108-119.

Valli, E., Ayyad, Z., Garcia-Salas, P., Cevoli, C., Afaneh, I. A., Bendini, A., et al. (2019). Influence of an innovative and promising gas clarification process $n$ the quality of stored extra virgin olive oils. Food Research International, 116, 30-36.

Venables, W. N., \& Ripley, B. D. (2002). Modern applied statistics with S (statistics and computing) (4th ed.). New York: Springer.

Wani, T. A., Masoodi, F. A., Gani, A., Nabi Baba, W., Rahmanian, N., Akhter, R., et al. (2018). Olive oil and its principal bioactive compound: Hydroxytyrosol - A review of the recent literature. Trends in Food Science \& Technology, 77, 77-90.

Xiang, C., Xu, Z., Liu, J., Li, T., Yang, Z., \& Ding, C. (2017). Quality, composition, and antioxidant activity of virgin olive oil from introduced varieties at Liangshan. $L W T$ Food Science and Technology, 78, 226-234.

Zago, L., Squeo, G., Bertoncini, E. I., Difonzo, G., \& Caponio, F. (2019). Chemical and sensory characterization of Brazilian virgin olive oils. Food Research International, 126, 108588. 